\title{
Evaluation of the effectiveness of virgin camel's urine as antifungal agents
}

\begin{abstract}
In the religion of Muslims, urine of the virgin camels is considered a method to treat some diseases. This has been studied with neutrality to confirm these beliefs. The controversy revolves around the effectiveness of the virgin camels urine in treating many diseases, as well as its positive role as inhibitors for the growth of fungi, as the inheritance and folk medicine among the inhabitants of the Arabian Peninsula confirm the effectiveness of the urine of the virgin camel as anti-fungi and this is denied by the international scientific community. Some research has been conducted in Saudi Arabia on the role of camel urine as anti-fungi, and therefore we have conducted some research here on proving the effectiveness of virgin camel urine as anti-fungal against Aspergillus niger and Epicoccum purpurascens and comparing that effectiveness with ewe and Human urine. In order to obtain fungi for conducting antagonistic experiments, fungi present in shower fibers (loofah) and dishwashing sponges were isolated. A. niger was isolated from all the bath fibrils (organic white or plastic), and no other fungi appeared, while the same fungus appeared in the bath sponge along with another fungus which is E. purpurascens. Results showed the efficacy of virgin camels urine in inhibiting growth of $A$. niger and E. purpurascens in liquid and solid media after a 7 days at $28^{\circ} \mathrm{C}$. There was no effective addition of ewes and human urine. Results corroborate the efficacy of the virgin camels urine to inhibit of growth of $A$. niger and E. purpurascens
\end{abstract}

Keywords: antifungal agent, Aspergillus niger, Epicoccum purpurascens, dishwashing sponge, shower bath fiber (loofah), virgin camels female urine.
Volume 8 Issue 4 - 2020

\author{
Hani MA Abdelzaher, 1,2 Mohamed \\ Alsuhaymi, ${ }^{3}$ Fawwaz MT Alshammari,' Senaid \\ AS Alshammari, I Zeyad MS Alshammari' \\ 'Department of Biology, Jouf University, Saudi Arabia \\ ${ }^{2}$ Department of Botany and Microbiology, Minia University, \\ Egypt \\ ${ }^{3}$ Department of Forensic Evidence, Forensic Laboratories, Saudi \\ Arabia
}

Correspondence: Hani MA Abdelzaher, Professor, Biology Department, College of Science, Jouf University, P.O. Box: 2014, Sakaka, Saudi Arabia, Tel +966- 558208457, Email hmdaher@ju.edu.sa

Received: October 31, 2020 | Published: November 13, 2020

\section{Introduction}

Fungi are, often, microorganisms that follow the Myceteae kingdom. Its spores live almost everywhere on the surface of the Earth, and only its colonies are seen that form after the growth of their spores when organic matter and moisture or hosts are available. Since fungi are non-autotrophic organisms, they obtain their food either by saprophyting on organic matters, parasitizing (on humans - animals plants - algae - other fungi), or by symbiosis with another organism from an elegant plant or algae. Many fungi can be isolated from soil, air, water, and wherever organic matter is found. The quality of the isolated fungi varies according to the type of soil and the percentage of moisture in it, as well as the type of crops grown or naturally grown in the soil. ${ }^{1}$ Virgin urine of camels (Camelus dromedaries L.) has become the topic of the hour among the inhabitants of the Arabian Peninsula and the subject vacillates between the inherited popular experiences and some few scientific studies that have dealt with this topic. Muslims dealt with the issue of treatment with milk and camel urine with certainty, documenting that with what was mentioned in their holy book (the Quran). ${ }^{2}$ For this purpose, we conducted a study on the effectiveness of virgin urine as an antagonist for some fungi isolated from a bath shower fiber (loofah) and a household dishwashing sponge.

Camel urine contains large amounts of magnesium, potassium, and albumin, as it contributes to the ability of camels to retain the sodium component in the body to prevent the exit of large quantities of water and to keep it inside the body for long periods. It is worth to mention that camels drink water only once during the winter season, and four times in the summer. It is worth noting that camels urine is used in the treatment of many different diseases in folk medicine or alternative medicine. The Arabs used to collect camels urine and boil it at high temperatures and then drink it for the treatment of some liver diseases. In some cases they dried camels urine on some types of herbs and used it to treat burns and some types of bleeding. Additionally, camels urine was used and mixed with camel's milk to treat some stomach diseases, dental pain, and some skin diseases. ${ }^{3}$ In the Arab Bedouin heritage as well, one of the uses of camel urine is that some women use it to wash their hair to lengthen it, give it a blonde and shine. They postulated that camels urine is effective in treating liver tumors and some diseases, such as boils, wounds that appear in the body, toothache and eye washing. ${ }^{4}$ A study conducted in Sudan (the country famous for breeding camels) showed that the therapeutic properties of camel urine was what he saw in the behavior of some tribe members who drank this urine when they had digestive disorders. He sought help from some doctors to study camel urine. Where they brought a group of patients and prescribed this urine to them for a period of two months, their bodies cleared of what they were suffering from. He further postulated that his study proved the benefit of camels urine in treating some diseases of the digestive system. ${ }^{5} \mathrm{He}$ added and pointed out that the best types of camels whose urine can be used in treatment are female virgin camels. In one of the master's thesis, the researcher succeeded in confirming the effectiveness of a preparation prepared from camel urine, which is the first antibiotic manufactured in this way in the world, and among the advantages of the product, as the researcher says: "It is inexpensive, easy to manufacture, and treats eczema, allergies, wounds, burns, acne, nail injuries, cancer, hepatitis and dropsy, with no collateral damage". ${ }^{6}$

It is worth noting that the benefits of camel urine in treating some of the aforementioned cases have not yet been scientifically proven. There is a disagreement about the use of camels urine for human treatment. Some people believe that alternative medicine has effective treatments that are worth studying and dealing with. Others argue that we should be wary of using folk remedies that studies have not proven the safety of their use, and among these folk remedies is camel urine. It has been mentioned above, some supporters for the benefits of the virgin urine of the camel. On the contrary, there are warnings 
from the World Health Organization (WHO) about the use of camel products, such as meat, milk, and urine, after the spread of the Middle East corona virus, which causes infection with what is known as Middle East Respiratory Syndrome. Therefore, care must be taken when using camel products to make sure that they are safe and do not contain any diseases or toxins and obtain them from reliable sources only. Some studies on the benefits of virgin camel urine, which the researcher Ahlam Al-Awadhi, conducted in conjunction with Dr. Nahed Haykal, at the College of Education for Girls in Jeddah, Saudi Arabia, and demonstrated the ability of these urine to eliminate $A$. niger that causes diseases for humans, animals and plants. ${ }^{2}$ To select fungi to experiment with the effect of virgin camel urine, fungi found in shower fiber and dishwashing sponges were selected in a number of homes. The reason for this choice is that many of the news in the recent period dealt with the colonization of a number of germs inside the bath fiber, and that is where constant moisture is provided, which is a suitable environment for the presence of fungi. The aim of this study is to isolate fungi present in the number of 10 shower bath fibers (loofah) used for bathing and a sponge for washing dishes, from 10 houses in Sakaka city, Al-Jouf, Saudi Arabia, and test the efficacy of urine of 2 virgin camels, 2 virgin ewes and a human on the growth of Aspergillus niger Van Tieghem and Epicoccum purpurascens Ehrenb. isolated from those sources.

\section{Materials and methods}

\section{Collection of fibrous samples from houses of Sakaka city and isolation of fungi}

Thirty Fibers ( 10 white for a shower +10 plastic for a shower +10 sponge for washing dishes) were collected from 10 different houses, in Sakaka city, Jouf, Saudi Arabia, in September - December 2019. Samples were placed in UV sterilized plastic bags, then inside the isolation cabinet, they were placed in (sterile) bottled water and shaken well, then kept in the refrigerator for 24 hours, until the process of cultivation on the cultural medium. One $\mathrm{ml}$ of the suspension of this water in the bottles (mentioned above) was placed in a sterile petri dish $(9 \mathrm{~cm})$, on which $15 \mathrm{ml}$ of the medium was poured (just before the solidification) suitable for growth of fungi and containing an inhibitor for bacterial growth (Rose Bengal-PDA), under aseptic conditions, next to the flame in the isolation cabinet, and left until it solidifies. Dishes were placed upside down, after being sealed with parafilm, in the incubator at $28^{\circ} \mathrm{C}$ for 7 days. After appearance of the fungal colonies, each colony (containing one fungal species) was transferred to a petri dish containing a sterile PDA medium (without rose-bangal), then the dishes were incubated again at $28^{\circ} \mathrm{C}$ for 7 days.

\section{Identification of isolated fungi}

Morphological (microscopic) identification of the isolated fungi was done with the help of the references of ${ }^{8,9}$ by using a compound microscope, in the Department of Biology, College of Science, Jouf University, and visualizing the samples, then the images were placed and arranged in a distinct plate for each type.

\section{Collecting camel urine samples + human urine + sheep urine}

Samples of virgin camels urine were collected from camels and sheep gatherings from of Al-Rousan area, Jouf, Saudi Arabia. Ewes and human urine were collected from the same area. All samples were obtained by placing them in sterile bottles under strict conditions to prevent contamination and were brought to the laboratory in a cool box.

\section{Freeze concentration of urine samples using freeze dryer (lyophilization)}

Every $100 \mathrm{ml}$ of tested urine is concentrated with a Freeze Dryer device to a volume of $20 \mathrm{ml}$.

\section{Effectiveness of urine of each virgin camels, virgin ewes and human on inhibiting growth of $A$. niger and E. purpurascens}

Ability of each type of urine (mentioned previously) to antagonize against $A$. niger and E. purpurascens was tested using the following two methods:

A. Contrast the two hardened media (PDA \& Sabouraud Dextrose Agar) by growing more than one inoculum for the same fungus in one Petri dish, containing each of the two above-mentioned nutrient media. Each plate was inlaid with three pits ( 3 wells) each containing $1 \mathrm{ml}$ of the freeze concentrated urine that passed through Millipore membrane (control of $1 \mathrm{ml}$ sterile distilled water), followed by parafilm sealing, then incubated at $28^{\circ} \mathrm{C}$ for 7 days.

B. Growth was carried out on PDbroth medium and subsequently the dry weight of the fungus was measured as an indication of growth. One hundred $\mathrm{ml}$ liquid medium was placed in each Erlenmeyer flask $(250 \mathrm{ml})$, and sterilization was done in an autoclave (at $121^{\circ} \mathrm{C}$, for a period of 20 minutes. After sterilization, $5 \mathrm{ml}$ of each type of tested freeze concentrated urine (control was $5 \mathrm{ml}$ sterile distilled water) were added. Three discs from each fungus (previously grown on PDA), were then added and incubated at $28^{\circ} \mathrm{C}$ for 7 days. Fungal dry weight was measured by placing filter papers in an electric oven $\left(85^{\circ} \mathrm{C}\right)$ for 24 hours, then weighing it after cooling in a desiccator. The growing fungus was filtered through filter paper and dry in the same oven at $\left(85^{\circ} \mathrm{C}\right)$ for another 24 hours, then weigh the filter paper containing the dry growth. Fungal dry weight was calculated by subtracting the weight of the filter paper from the weight of the dry growth + filter paper.

\section{Statistical analysis}

Difference in data from the control (in the experiment measuring the effect of urine on mycelial growth of the two tested fungi was treated using a t-test in the Waller-Duncan $\mathrm{K}$ ratio (with some modifications), which shows the significant differences expressed for each of $(\mathrm{P}<0.05 \leqq \mathrm{P}<0.01 \leqq \mathrm{P}<0.001) .{ }^{10}$ All results were an outcome of an average of three readings, and each trial was repeated at least twice.

\section{Results}

\section{Identification of isolated fungi}

A. niger was isolated and identified only from all the fibers used for showering and even the dishwashing sponge, while E. purpurascens and $A$. niger were isolated from the dishwashing sponge. The two fungi were identified using the previously mentioned identification keys, and the genera of the two isolated fungal species occurred under the following two genera:

I. The filamentous Ascomycetes of the genus Aspergillus, one species was known, which was $A$. niger (Figure $1 \&$ Figure 2).

II. The filamentous Ascomycetes of the genus Epicoccum which was E. purpurascens (Figure $1 \&$ Figure 3). 

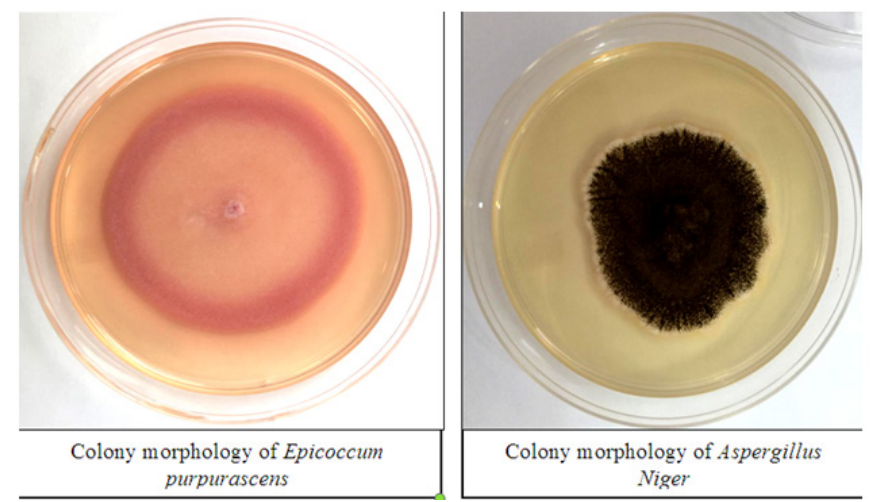

Figure I Colony morphology of A. niger and E. purpurascens on PDA medium after 7 days at $27^{\circ} \mathrm{C}$ in the dark.
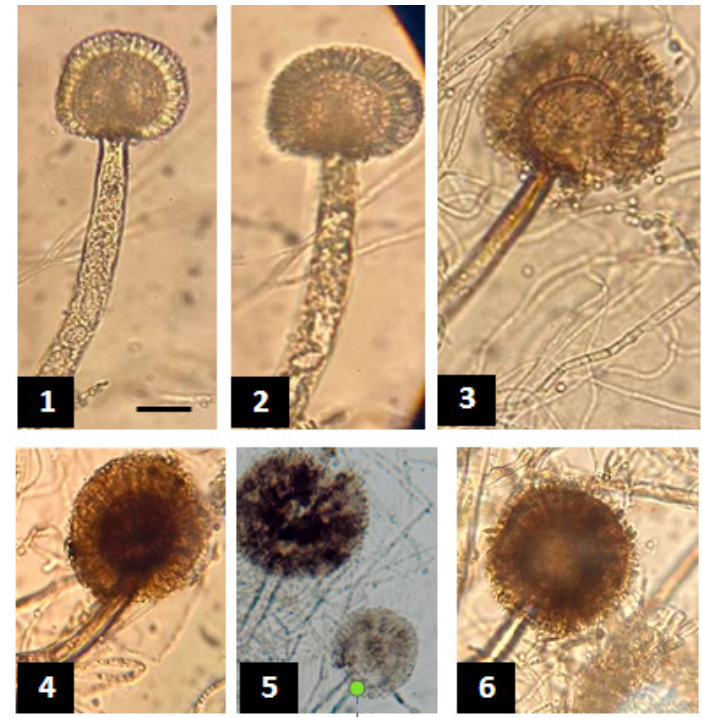

Figure 2 I-6: Growth of Aspergillus niger under a compound microscope. A thick, aseptate conidiophore with radially arranged symmetrical stergmata. Bar $10 \mu \mathrm{m}$ in image (I) is the same as for the rest of the photos.
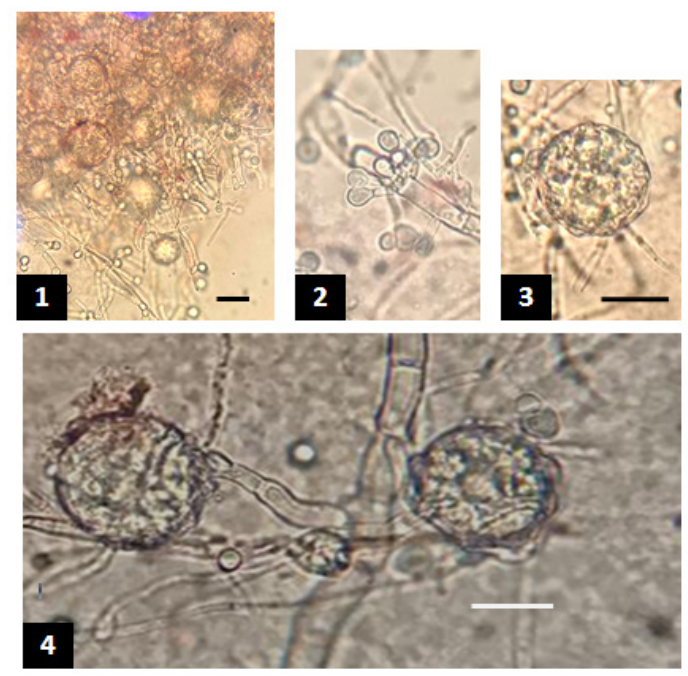

Figure 3 Growth of Epicoccum purpurascens under a compound microscope. I-4:Thick septate conidiophores with spherical granulated conidia. Bar 10 $\mu \mathrm{m}$ in picture $(\mathrm{I})$ is for this image only, in image 3 it is also for image 2, and in image 4 it is for this image only.
Results show the presence of $A$. niger in all samples of the fibers (loofah) used in bathing, whether it was white loofah or plastic ones, and even a dishwashing sponge. E. purpurascens appeared in dishwashing sponge only and did not appear in the bathing sheets (Table 1).

\section{Ability of camels virgin urine, ewe's urine and human urine to antagonism against $A$. niger and E. purpurascens on PDA and Sabouraud dextrose agar media}

Results indicate the effectiveness of urine of the camel No. (1) and No. (2) in inhibiting growth of $A$. niger in areas where fungal growth meets wells containing urine, after 7-days at of $28^{\circ} \mathrm{C}$, in the dark (Figure 4). Results also show the effectiveness of each of the virgin camels urine No. (1) and No. (2) in inhibiting growth of E. purpurascens in areas where the fungal growth meets the wells containing urine, after 7 days $28^{\circ} \mathrm{C}$, In the dark (Figure 5).
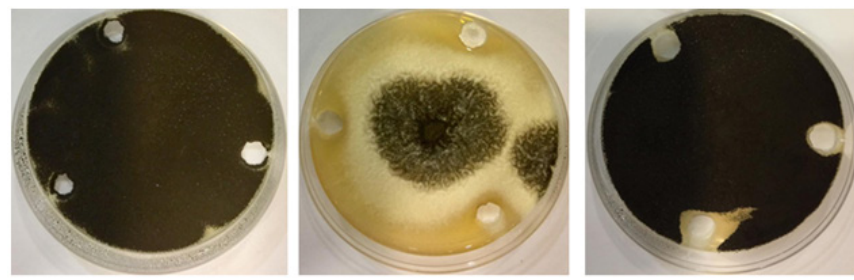

Figure 4 Efficacy of virgin camels urine towards Aspergillus niger; left of the images (control - sterile distilled water only), in the middle of the images is the effect of camels urine No. (I), the right of the images is the effect of camels urine No. (2), on Sabouraud Dextrose medium, after a week at $28^{\circ} \mathrm{C}$.
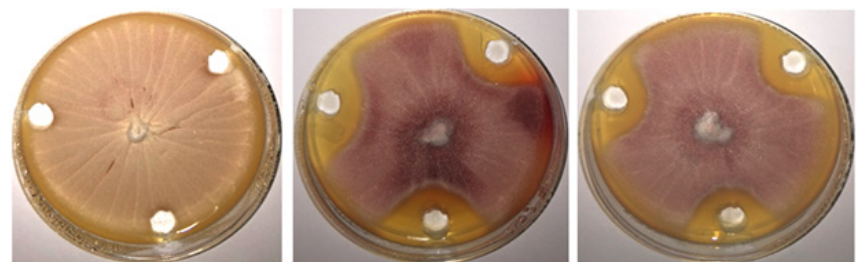

Figure 5 Efficacy of virgin camels urine towards E. purpurascens; left of the images (control - sterile distilled water only), in the middle of the images is the effect of camels urine No. (I), the right of the images is the effect of camels urine No. (2), on Sabouraud Dextrose.
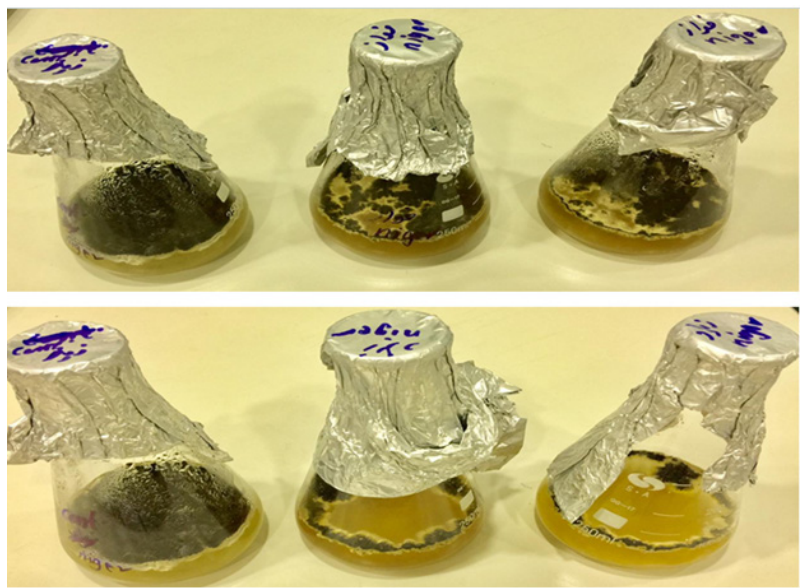

Figure 6 Effectiveness of the urine of a camels (grouped above, animal No. (I) towards growth of A. niger, left of the pictures (control - distilled water), in the middle and the right of the pictures is the effect of urine. Grouped at the bottom, animal No. (2) towards growth of A. niger, left of the images (Control - Distilled water), in the middle and the right are the effect of urine. 


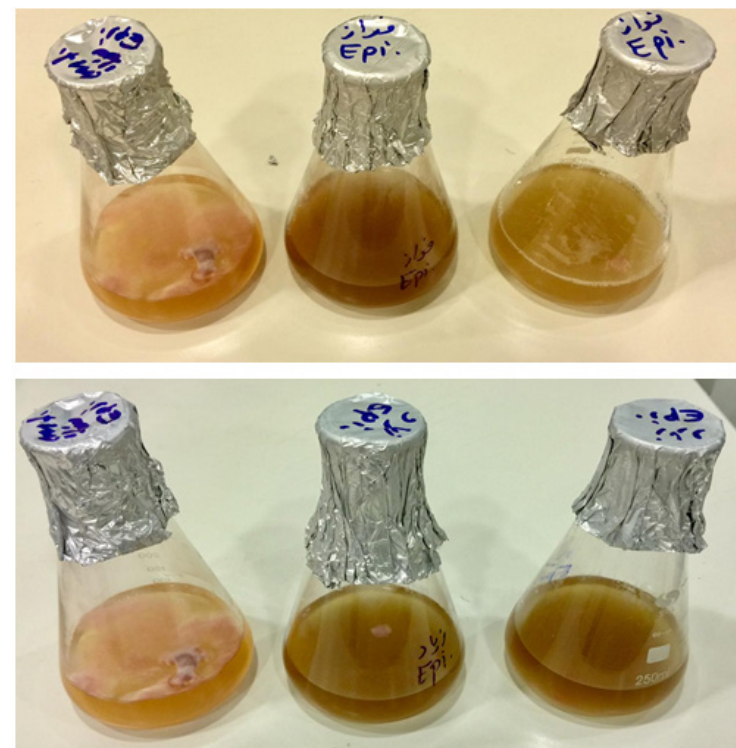

Figure 7 Effectiveness of the urine of a camels (grouped above, animal No. (I) towards growth of E. purpurascens, left of the pictures (control - distilled water), in the middle and the right of the pictures is the effect of urine. Grouped at the bottom, animal No. (2) towards growth of E. purpurascens, left of the images (Control - Distilled water), in the middle and the right are the effect of urine.

\section{Ability of camels virgin urine, ewe's urine and human urine to antagonism against $A$. niger and E. purpurascens on PDbroth medium}

Results demonstrate the effectiveness of both virgin camel urine No. (1) and No. (2) on reducing the dry weight of $A$. niger grown in PDbroth medium after 7 days incubation at $28^{\circ} \mathrm{C}$ in the dark (Figure 6). Results also appear the effectiveness of urine of the female virgin camel No. (1) and No. (2) On inhibiting the growth of E. purpurascens (dry weight in flasks of PDbroth medium containing tested urine), after 7 days incubation at $28^{\circ} \mathrm{C}$ in the dark (Figure 7). The following table shows dry weight of the two fungi after treating them with the urine of each of (two females of the virgin camel + ewe + human), as it shows a very strong significant effect, inhibiting the camel's urine towards each of the two fungi, but the effect is more powerful on $E$. purpurascens compared to $A$. niger. There is no remarkable effect on the ewe and human urine (Table 2 ).

Table I Fungi isolated from different fibrils from 5 houses in Sakaka city

\begin{tabular}{clll}
\hline No. & $\begin{array}{l}\text { Place of } \\
\text { sampling }\end{array}$ & $\begin{array}{l}\text { Type of bathing } \\
\text { fiber (loofah) }\end{array}$ & Isolated fungi \\
\hline I & $\begin{array}{l}\text { Houses No. } \\
(I-10)\end{array}$ & $\begin{array}{l}\text { White plant fibers } \\
\text { Plastic }\end{array}$ & Aspergillus niger \\
5 & $\begin{array}{l}\text { House No. } \\
(I-10)\end{array}$ & $\begin{array}{l}\text { Dishwashing } \\
\text { sponge }\end{array}$ & $\begin{array}{l}\text { Aspergillus niger } \\
\text { Epicoccum purpurascens }\end{array}$ \\
\hline
\end{tabular}

Table 2 Effect of urine (female virgin camel - ewe - human) ( $5 \mathrm{ml}$ concentrated solution by dryer freezing in $10 \mathrm{ml}$ medium) on the growth of $A$. niger and $E$ purpurascens in PDbroth medium (expressing growth by dry weight) After 7 days, at $28^{\circ} \mathrm{C}$

\begin{tabular}{llll}
\hline No. & $\begin{array}{l}\text { Urine } \\
\text { source }\end{array}$ & $\begin{array}{l}\text { Aspergillus niger } \\
\text { dry weight }(\mathbf{g m})\end{array}$ & $\begin{array}{l}\text { Epicoccum purpurascens } \\
\text { dry weight } \mathbf{( g m )}\end{array}$ \\
\hline & $\begin{array}{l}\text { Control } \\
\text { (sterile }\end{array}$ & $0.378 \pm 0.00$ I & $0.208 \pm 0.003$ \\
\hline $\begin{array}{l}\text { distilled } \\
\text { water) }\end{array}$ & & \\
\hline
\end{tabular}

Table continue

\begin{tabular}{llll}
\hline No. & $\begin{array}{l}\text { Urine } \\
\text { source }\end{array}$ & $\begin{array}{l}\text { Aspergillus niger } \\
\text { dry weight }(\mathbf{g m})\end{array}$ & $\begin{array}{l}\text { Epicoccum purpurascens } \\
\text { dry weight }(\mathbf{g m})\end{array}$ \\
\hline $\mathbf{2}$ & $\begin{array}{l}\text { Virgin } \\
\text { camel (I) }\end{array}$ & $0.015^{\circ} \pm 0.003^{* * *}$ & $\begin{array}{l}0.000^{c} \pm 0.000^{* * * *} \text { (No } \\
\text { growth) }\end{array}$ \\
$\mathbf{3}$ & $\begin{array}{l}\text { Virgin } \\
\text { camel (2) }\end{array}$ & $0.138 \pm 0.005^{* *}$ & $\begin{array}{l}0.000 \pm 0.000^{* * *} \text { (No } \\
\text { growth) }\end{array}$ \\
$\mathbf{4}$ & $\begin{array}{l}\text { Virgin ewe } \\
(I)\end{array}$ & $0.328 \pm 0.007$ & $0.188 \pm 0.007$ \\
$\mathbf{5}$ & $\begin{array}{l}\text { Virgin ewe } \\
(2)\end{array}$ & $0.320 \pm 0.008$ & $0.191 \pm 0.009$ \\
$\mathbf{6}$ & Human & $0.369 \pm 0.003$ & $0.194 \pm 0.006$
\end{tabular}

$\mathrm{c}$ is the mean of three measurements followed by: $*$ significant at $\mathrm{p}<0.05$, ** $=$ highly significant at $\mathrm{p}<0.0 \mathrm{I}, * * *=$ very high significant at $\mathrm{p}<0.00 \mathrm{I}$, according to Waller-Duncan K-ratio t- test (with some modification).

\section{Discussion}

Many medical sources warn against the flourishing of microbial germs in the bathing loaf, due to the constant availability of moisture in the fibers, which constitutes continuous sources for the transfer of germs to the bodies of those dealing with these means, especially those people who are immunocompromised and who have allergies. We are informed by successive news in the media and social media about the dangers of using a shower loofah, even though many of us use these fibers constantly and almost daily. ${ }^{11}$ In this research, $A$. niger was isolated from all the fibers that were studied, even a sponge used for washing dishes, and this indicates the ability of this fungus to exist between the folds of these fibrils, whether natural or plastic, in a moist environment exposed to many detergents and shower soap. This is in line with a lot of research showing the presence of this fungus in humid environments. ${ }^{12,13}$ E. purpurascens was isolated from a dishwashing sponge only, while it was not isolated from the shower loofah, and this may be attributed to the suitability of the dishwashing sponge environment on the presence of this fungus. ${ }^{14}$ It is worth noting that $A$. niger which was isolated from all the fibers and sponges in this study, produced huge quantities of conidia and is considered one of the largest causes of very serious diseases similar to tuberculosis and called Aspergillosis, which has been proven by many previous studies. Subsequently, with daily and consecutive use of household dishwashing sponges containing fungi is considered a dangerous source to users who are exposed to contact with these fungi, which may cause many diseases, especially for the elderly and immunocompromised and children. One recent study reported that Epicoccum (isolated in this study from a dishwashing sponge) is one of the most important fungal allergens. It was responsible for causing many respiratory allergic disorders, such as upper and lower respiratory tract diseases, rhinitis, sinusitis, and asthma. High density of conidia of Epicoccum may trigger or exacerbate asthma attacks in children. ${ }^{15}$ Epicoccum have been reported to be the cause of severe allergic disorders, including hypersensitivity pneumonitis and allergic fungal sinusitis, in 5-7\% of the population worldwide. ${ }^{16}$ Therefore, the presence of this instinct is considered to be an allergen.

As a result of the many diseases that fungi cause to humans, the pace has accelerated towards the search for sources to resist these microorganisms and reduce their risks. Scientific research has continued towards the use of antifungals, especially those with natural sources that do not cause pollution problems to the environment surrounding the human being. Urine of the virgin camel is considered one of the natural sources and has a historical reputation among the inhabitants of the Arabian peninsula in controlling fungi. Unfortunately modern science does not recognize benefits of that urine, rather the World Health Organization has warned against the use of camel meat and 
products for fear of transmitting the Corona virus that is endemic to camels (WHO warning of using camels productions). On the contrary, some studies in some islamic countries dealt with the positive role of camel urine (especially virgin camel) in treating many diseases, including fungal diseases for humans. Therefore, we had to conduct some experimental research on a scientific basis in an attempt to prove the effectiveness of the virgin camels urine towards controlling some fungi. Therefore, two fungi of A. niger and E. purpurascens isolated from bath fibrils and a dishwashing sponge were chosen to conduct fungi antagonistic research, with a comparison of virgin camels urine with ewes and human urine. The study here proved that the urine of virgin camels that were grazing in the Nafud desert in the Sakaka region, Saudi Arabia, has the ability to inhibit the growth of $A$. niger and E. purpurascens in both the solidified and the liquid nutrient media and this is consistent with one of the studies which was conducted in the region of Jeddah, Saudi Arabia. ${ }^{2}$ From here it can be said that the urine of a virgin camel has a great inhibitory role for the growth of $A$. niger and E. purpurascens, and that urine can be used in one way or another to control these fungi.

We recommend further studies on:

a) The role of more of the camel strains as antibiotics for all sections of the different fungi.

b) Determining the type of active substances in the urine of the camel to find anti-fungal preparations or drugs.

c) Conducting complementary studies on the application of that antagonism in the methods of biological control of plant diseases.

d) Soak bath wipes (loofah) and dishwashing sponges in disinfectant solutions when not in use, to reduce the presence and flourishing of fungal conidia and spores to prevent their transmission to people and animals with successive use.

\section{Acknowledgements}

The authors thanks to Biology Department, College of Science, Jouf University and Al-Jouf Region Police, Forensic Laboratories, Forensic Evidence Department, Sakaka, Al-Jouf, Saudi Arabia for their financial and moral support towards the completion and emergence of this study.

\section{Conflicts of interest}

The author declares that there is no conflict of interest.

\section{References}

1. Abdelzaher HMA, Moustafa SMN, Al-Sheikh H, et al. The genus Pythium in three different continents. Pythium: Dignosis Diseases and Management. Taylor and Francis; 2020.

2. Abdalall AHA. The inhibitory effect of camel's urine on mycotoxins and fungal growth. Afri J of Agric Res. 2010;5(11):1331-1337.

3. Al-Attas AS. Determination of Essential Elements in Milk and Urine of Camel and in Nigella sativa Seeds. Arab J Chem. 2008;1(2):123-129.

4. Thakur AN. Therapeutic use of urine in early Indian medicine. Ind J. Hist Sci. 2004;39(4):415-427.

5. Muhammad UMAD. A study of chemical components and some medicinal uses of Arabian camel urine. Sudan. University of Gezira; 1998.

6. Al-Qattan M. An antibiotic from camel urine. Saudi Arabia. College of Education for Girls; 1998.

7. Moustafa SMN, Abdelzaher, HMA. Occurrence of Hemolytic Fungi Mounted on Wheat Grains in the main Silo of Sakaka, Saudi Arabia. $J$ OF PURE AND APPL MIC. 2016;10(3):1817-1824.

8. Ainsworth GC, Bisby GR. Ainsworth \& Bisby's dictionary of the fungi. Commonwealth Mycological Institute; 1971.

9. Ainsworth GC, Bisby GR, Kirk PM, et al. Ainsworth \& Bisby's dictionary of the fungi. In: Kirk PM, editor. Wallingford, Oxon, UK. CABI Pub; 2001.

10. Waller RA, Duncan DB. A Bayes Rule for the Symmetric Multiple Comparison Problem. J Americ Statis Assoc. 1969;64:1484-1504.

11. Anaissie EJ. Cleaning patient shower facilities: A novel approach to reducing patient exposure to aerosolized Aspergillus species and other opportunistic molds. Clin Infect Dis. 2002;35:86-88.

12. Shehu K, Bello MT. Effect of Environmental Factors on the Growth of Aspergillus Species Associated with Stored Millet Grains in Sokoto. Nig $J$ of Basic and Appl Sci. 2011;19(2):218-223.

13. Shaista $\mathrm{P}$, Lanjewar $\mathrm{S}$, Sharma $\mathrm{K}$, et al. Isolation of fungi from the surface water of river. J Exp Sci. 2011;2(10):58-59.

14. Nontokozo M, Magwaza EN, Bhekie B, et al. The occurrence and diversity of waterborne fungi in African aquatic systems: Their impact on water quality and human health. Int J Environ Res Public Health. 2017:546.

15. Halewyn M, Chevalier P. Epicoccum purpurascens. 2019.

16. Flannigan B, Samson RA, Miller JD. Microorganisms in home and indoor work environments: diversity, health impacts, investigation and control. Boca Raton. CRC Press; 2011:147-182. 